\title{
Surface electronic structure of bismuth oxychalcogenides
}

\author{
S. V. Eremeev $\oplus^{1,2,3}$ Y. M. Koroteev, ${ }^{1,2,3}$ and E. V. Chulkov ${ }^{4,2,3,5,6}$ \\ ${ }^{1}$ Institute of Strength Physics and Materials Science, 634055 Tomsk, Russia \\ ${ }^{2}$ Tomsk State University, Laboratory of Nanostructured Surfaces and Coatings, 634050 Tomsk, Russia \\ ${ }^{3}$ Saint Petersburg State University, Laboratory of Electronic and Spin Structure of Nanosystems, 198504 Saint Petersburg, Russia \\ ${ }^{4}$ Centro de Física de Materiales-MPC and Centro Mixto, CSIC-UPV/EHU, 20018 San Sebastián/Donostia, Spain \\ ${ }^{5}$ Donostia International Physics Center, 20018 San Sebastián/Donostia, Spain \\ ${ }^{6}$ Departamento de Física de Materiales, UPV/EHU, Facultad de Ciencias Químicas, UPV/EHU, \\ Apartado 1072, 20080 San Sebastián/Donostia, Spain
}

(Received 3 June 2019; revised manuscript received 2 August 2019; published 12 September 2019)

\begin{abstract}
Within density functional theory we study the bulk band structure and surface states of bismuth oxychalcogenides $\mathrm{Bi}_{2} \mathrm{O}_{2} \mathrm{Se}$ and $\mathrm{Bi}_{2} \mathrm{O}_{2}$ Te. We consider both polar and nonpolar surface terminations. On the basis of relativistic $a b$ initio calculations, we show that both unreconstructed (polar) and reconstructed (nonpolar) surfaces possess the Rashba spin-split surface states. The metallic Rashba-split states on polar surfaces stem from huge potential bending, positive or negative, depending on surface polarity. On the nonpolar surfaces resulting from single-crystal cleavage the emerging Rashba-split states are nonmetallic.
\end{abstract}

DOI: 10.1103/PhysRevB.100.115417

\section{INTRODUCTION}

Recently, materials with a strong spin-orbit interaction were intensively studied [1]. A feature of this interaction is that it can lead to a spin splitting of electronic states even in nonmagnetic systems, which opens up the possibility of manipulating the spin degrees of freedom of carriers without using an external magnetic field. The spin-orbit interaction lifts the degeneracy of the electronic states for electrons moving in two-dimensional (2D) geometries without inversion symmetry that is a Rashba effect. The discovery of a large Rashba splitting of two-dimensional electron states on the Bi surfaces and $\mathrm{Bi}$-induced $2 \mathrm{D}$ surface alloys on noble metals [2-6] gave impetus to the search for semiconductor systems with large spin splitting. In particular, the study of a polar semiconductors $\mathrm{BiTe} X(X=\mathrm{Cl}, \mathrm{Br}, \mathrm{I})$ demonstrated the giant Rashba-type spin splitting of its bulk states [7-10] as well as of the surface states [8,10-19].

The spin-orbit interaction is also responsible for the appearance of a topological insulator phase [20-22], characterized by an insulating bulk gap and gapless Dirac-type surface states. In contrast to the Rashba states, the surface electronic structure in topological insulators can be viewed as if the two branches have opposite dispersions and touch at a single point, i.e., at the Dirac point. The topological phase can be achieved in inversely stacked, centrosymmetric $\operatorname{BiTe} X$ and their thin films $[23,24]$ and in BiTe $X$ under external pressure $[25,26]$ or via temperature-induced structure transformation [27]. In the latter case it was demonstrated that annealing of BiTeI leads to transformation into the $\mathrm{Bi}_{2} \mathrm{Te}_{2} \mathrm{I}$ phase, similar to the layered hexagonal, tetradymitelike compounds $\mathrm{Bi}_{2} \mathrm{Te}_{3}$ and $\mathrm{Bi}_{2} \mathrm{Se}_{3}$ (space group $R \overline{3} m$ ), which currently are the most studied, both experimentally [28-31] and theoretically [32-35], three-dimensional topological insulators. Ternary tetradymites, such as $\mathrm{Bi}_{2} \mathrm{Te}_{2} \mathrm{~S}, \mathrm{Bi}_{2} \mathrm{Te}_{2} \mathrm{Se}$,
$\mathrm{Bi}_{2} \mathrm{Se}_{2} \mathrm{Te}$, and $\mathrm{Bi}_{2} \mathrm{Se}_{2} \mathrm{~S}$, also crystallize in space group $R \overline{3} m$ and possess the topological insulator phase [36-40], while other compounds of the $\mathrm{Bi}_{2} X_{2} Y$ family $(X, Y=\mathrm{O}, \mathrm{S}$, Se, and $\mathrm{Te}$ ) can crystallize in different structures. For example, $\mathrm{Bi}_{2} \mathrm{~S}_{2} \mathrm{Se}$ has orthorhombic crystal structure with space group Pnma [41], and the $\mathrm{Bi}_{2} \mathrm{O}_{2} \mathrm{~S}$ crystal has both orthorhombic and tetragonal structures with space group symmetries Pnnm and $P 4 / \mathrm{nmm}$, respectively [42]. $\mathrm{Bi}_{2} \mathrm{O}_{2} \mathrm{Se}$ and $\mathrm{Bi}_{2} \mathrm{O}_{2} \mathrm{Te}$ also present a tetragonal-type lattice but with space group symmetry $I 4 / \mathrm{mmm}$ [43-47].

Earlier studies of $\mathrm{Bi}_{2} \mathrm{O}_{2} \mathrm{Ch}$ (Ch is a chalcogen atom, Te or $\mathrm{Se}$ ) were mainly focused on their transport and thermoelectric properties [44,48-52]. Recently, successful growth of thin and ultrathin $\mathrm{Bi}_{2} \mathrm{O}_{2}$ Se nanofilms [53-55] was reported. These nanofilms demonstrate such unique properties as very low thermal conductivity and high electrical conductivity [49], as well as ultrahigh Hall mobility [54]. Additionally, $\mathrm{Bi}_{2} \mathrm{O}_{2} \mathrm{Se}$ may be used as an optical switch for the exploitation of compact and high-performance midinfrared pulsed laser sources [56]. In Reference [57] was found a strong reduction in amplitude of the universal conductance fluctuations and showed that this result agrees with the assumed strong spin-orbit interaction in the $\mathrm{Bi}_{2} \mathrm{O}_{2} \mathrm{Se}$ nanoplate. The strong spin-orbit interaction in $\mathrm{Bi}_{2} \mathrm{O}_{2} \mathrm{Se}$ nanoplates has also been confirmed through magnetotransport measurements [55]. The creation on the basis of these $\mathrm{Bi}_{2} \mathrm{O}_{2} \mathrm{Se}$ nanolayers of a highperformance and low-power transistor [58], supersensitive 2D phototransistors [59], the top-gate field-effect transistor [53], and a high-performing infrared photodetector [60] and the possibility of integrating high-quality $\mathrm{Bi}_{2} \mathrm{O}_{2} \mathrm{Se}$ with the silicon complementary metal-oxide-semiconductor technologies [59] have also been reported.

There are many studies in which high-resolution transmission electron microscopy (HRTEM) [53-55,58], x-ray photoelectron spectroscopy [54], x-ray diffraction [53], the 
method of selected area electron diffraction $[53,58]$, and energy-dispersive $\mathrm{x}$-ray spectroscopy $[53,59]$ were used to study the atomic structure of the $\mathrm{Bi}_{2} \mathrm{O}_{2} \mathrm{Se}(001)$ nanofilms synthesized via a facial chemical vapor deposition method. These films have shown excellent controllability of thickness, domain size, nucleation site, and crystal-phase evolution. It was found that the films have atomically smooth surfaces, the morphology and roughness of which remain almost unchanged, even after exposure to air for several months, which indicates their excellent resistance to the environment. Dissimilar results were obtained for a $\mathrm{Bi}_{2} \mathrm{O}_{2} \mathrm{Se}(001)$ surface prepared by cleaving the bulk crystal inside the ultrahighvacuum chamber [61]. Due to the peculiarity of the $\mathrm{Bi}_{2} \mathrm{O}_{2} \mathrm{Se}$ crystal structure, where the $\mathrm{Se}$ atomic plane lies between two $\mathrm{Bi}-\mathrm{O}-\mathrm{Bi}$ trilayers and there is weak interaction between $\mathrm{Bi}$ and Se planes, the crystal cleaves in such a way that $50 \%$ of the Se atoms are attached to each Bi plane because of the electroneutrality requirement for the arising surfaces that leads to the appearance of complicated surface reconstruction [61].

To date, there are only two studies, Refs. [53,61], devoted to angle-resolved photoemission spectroscopy (ARPES) measurements, and both relate only to a $\mathrm{Bi}_{2} \mathrm{O}_{2}$ Se cleaved crystal (001) surface. The electron and hole pockets near the conduction band minimum (CBM) and valence band maximum (VBM), which are essential for the transport properties of $n$ - and $p$-type $\mathrm{Bi}_{2} \mathrm{O}_{2} \mathrm{Se}$, were studied in detail. These measurements, however, did not reveal the surface states on the cleavage surface. The electronic structure of the atomically smooth surfaces of the nanofilms has not been studied.

In this paper, using $a b$ initio electronic structure calculations, we examine the surface states of the $\mathrm{Bi}_{2} \mathrm{O}_{2} \mathrm{Se}$ and $\mathrm{Bi}_{2} \mathrm{O}_{2} \mathrm{Te}$ compounds for both the nonreconstructed surface geometry, as realized by chemical vapor deposition, and the reconstructed surface geometry, which occurs on cleavage surfaces of single crystals. We show that both types of the surface hold the Rashba spin-split surface states whose characteristic features depend on the polarity of the surface and $C h$ species.

\section{METHODS}

The density functional theory calculations were performed by using the Vienna Ab initio Simulation Package (VASP) $[62,63]$, with core electrons represented by projector augmented wave (PAW) potentials [64,65]. At the starting point we used the generalized gradient approximation of Perdew-Burke-Ernzerhof functional (GGA-PBE) [66] for the exchange-correlation potential. DFT-D3 van der Waals correction [67] was applied for structure optimization. To obtain accurate bulk band structures, the modified Becke-Johnson $(\mathrm{mBJ})$ exchange potential [68,69], which has been shown to be the most accurate semilocal potential for band gap calculations, and the HSE06 screened hybrid functional [70] were adopted. Spin-orbit interaction (SOI) was included in all types of calculations.

For surface band structure calculations we use the slab model and apply the DFT-1/2 self-energy correction method [71,72], which requires only the addition of a self-energy correction potential, calculated from a half-ionized free atom, to the standard DFT potential (PAW-PBE in our case). This method yields accurate band structures for many semiconductors, and owing to its low computational cost, DFT-1/2 can be considered as a good alternative to very expensive hybrid functional methods for systems that require large computational cells and for $\mathrm{mBJ}$ potentials, which diverge for surface calculations. Before using the DFT-1/2 method for surface calculation we compared its results for the bulk with $\mathrm{mBJ}$ and HSE06 spectra.

Symmetric Bi- (chalcogen-) terminated slabs with 39(41-) layer thickness were used for unreconstructed surfaces, and for $4 \times 1$ reconstructed nonpolar surfaces slabs with 33 atomic layers were utilized. The slabs were relaxed within the PBE + DFT-D3 approach to a depth of five atomic layers, whereas atoms in the internal layers were fixed to their bulk positions.

\section{RESULTS AND DISCUSSION}

\section{A. Bulk band structure}

$\mathrm{Bi}_{2} \mathrm{O}_{2} \mathrm{Ch}$ crystallizes into a body-centered tetragonal structure $(I 4 / \mathrm{mmm}$, No. 139) which has ten atoms in the unit cell with eight atomic layers, as illustrated in Fig. 1(a). Ch and O atoms occupy the $2 a$ and $4 d$ Wyckoff positions, respectively. The only free internal structure parameter of the tetragonal unit cell of $\mathrm{Bi}_{2} \mathrm{O}_{2} C h$ is $z_{B i}$, which determines the $4 e$ positions of the $\mathrm{Bi}$ atoms.

Bulk lattice parameters and $\mathrm{Bi}$ atomic positions of $\mathrm{Bi}_{2} \mathrm{O}_{2} \mathrm{Te}$ and $\mathrm{Bi}_{2} \mathrm{O}_{2} \mathrm{Se}$ were optimized. The optimized $a$ and $c$ parameters for $\mathrm{Bi}_{2} \mathrm{O}_{2}$ Te bulk have been found to be 3.979 and $12.769 \AA$, respectively, and they are 3.893 and $12.209 \AA$ for $\mathrm{Bi}_{2} \mathrm{O}_{2} \mathrm{Se}$. These parameters are in excellent agreement with experimental values $a=3.983(1), c=12.699(2) \AA$ for $\mathrm{Bi}_{2} \mathrm{O}_{2} \mathrm{Te}$ and $a=3.887(1), c=12.164(2) \AA$ for $\mathrm{Bi}_{2} \mathrm{O}_{2} \mathrm{Se}$ [45]. The optimized $z_{B i}$ was found to be equal to 0.347823 and 0.354665 for $\mathrm{Bi}_{2} \mathrm{O}_{2} \mathrm{Te}$ and $\mathrm{Bi}_{2} \mathrm{O}_{2} \mathrm{Se}$, respectively. These values are also in fine agreement with the experimental position $z_{B i}=0.3480(1)$ for $\mathrm{Bi}_{2} \mathrm{O}_{2} \mathrm{Te}$ and $0.35340(4)$ for $\mathrm{Bi}_{2} \mathrm{O}_{2} \mathrm{Se}$ [45].

The bulk electronic band structure of $\mathrm{Bi}_{2} \mathrm{O}_{2} \mathrm{Se}$ has been studied more than that of $\mathrm{Bi}_{2} \mathrm{O}_{2} \mathrm{Te}$. The indirect $(\mathrm{X}-\Gamma)$ band gap of $\mathrm{Bi}_{2} \mathrm{O}_{2}$ Se has been determined by ARPES to be around $0.8 \mathrm{eV}[53,61]$, and it was found to be $0.85 \pm 0.05 \mathrm{eV}$ from the scanning tunneling spectroscopy (STS) measurements [61]. The direct optical absorption band gap was estimated to be equal to $1.486 \mathrm{eV}$ [74].

For $\mathrm{Bi}_{2} \mathrm{O}_{2} \mathrm{Se}$ bulk [Fig. 1(b)] our PBE calculation yields a $\Gamma$ gap of $747 \mathrm{meV}$, while the indirect gap determined by the valence band (VB) maximum at the R point (energy of the VB top at $\mathrm{X}$ is about $1 \mathrm{meV}$ lower) and conduction band (CB) minimum lying at the $\Gamma$ point equals $335 \mathrm{meV}$. Both the $\mathrm{mBJ}$ and HSE06 calculations substantially increase the gap. The $\mathrm{mBJ}$ spectrum demonstrates an indirect $\mathrm{R}-\Gamma$ gap of $954 \mathrm{meV}$, and it is $931 \mathrm{meV}$ in the HSE06 calculation. In general, with the exception of VB maxima at the $\mathrm{R}$ and $\mathrm{X}$ points and CB minima at the $\Gamma$ and $\mathrm{M}$ points the HSE06 approach gives systematically, by $90-200 \mathrm{meV}$, lower (higher) energies for electronic bands of the VB (CB) compared to the $\mathrm{mBJ}$ calculation. While the obtained $\mathrm{mBJ}$ and HSE06 values of the indirect gap slightly overestimate the ARPES gap, 

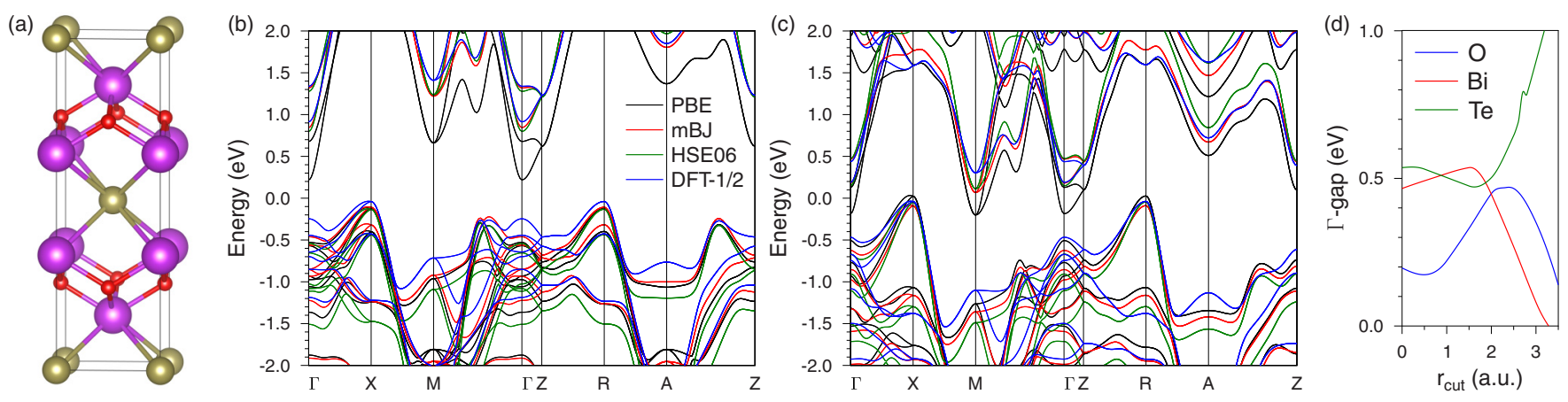

FIG. 1. (a) Atomic structure of the $\mathrm{Bi}_{2} \mathrm{O}_{2}$ Ch compounds (violet and red balls correspond to $\mathrm{Bi}$ and $\mathrm{O}$ atoms, respectively; khaki balls stand for $\mathrm{Ch}=\mathrm{Se}$, Te atoms). Here and throughout ball-and-stick atomic structures were created with VESTA [73]. Bulk electronic structure of (b) $\mathrm{Bi}_{2} \mathrm{O}_{2} \mathrm{Se}$ and (c) $\mathrm{Bi}_{2} \mathrm{O}_{2}$ Te calculated within the PBE, mBJ, HSE06, and DFT-1/2 approaches. (d) Dependence of the $\Gamma$ gap on $r_{\text {cut }}$ for the atomic self-energy potential $V_{S}$ in $\mathrm{Bi}_{2} \mathrm{O}_{2}$ Te.

they are in better agreement with the STS result. The direct $(\Gamma-\Gamma)$ gap values, $1.307 \mathrm{eV}(\mathrm{mBJ})$ and $1.340 \mathrm{eV}$ (HSE06), are in reasonable agreement with the optical absorption band gap. Earlier calculations performed within the $\mathrm{mBJ}$ approach $[51,53,75,76]$ demonstrated the gap varying from $0.85 \mathrm{eV}$ [53] to $1.28 \mathrm{eV}$ [51]. The larger value was obtained when the van der Waals $(\mathrm{vdW})$ corrections were not taken into account during the structure optimization and hence the lattice parameters (especially $c$ ) were overestimated. The SOI has not been taken into account either. When the SOI and vdW corrections were properly accounted for, the lattice parameters were found to be very close to our values (e.g., $a=3.892 \AA$ and $c=12.186 \AA$ in Ref. [75]), and the mBJ gap (1.092 eV [75]) got close to our result $(0.954 \mathrm{eV})$. The small difference can be apparently attributed to different $z_{B i}$ (which was not reported in Ref. [75] and in many other works). The HSE06 results reported gaps of $0.89 \mathrm{eV}$ [77] and $0.99 \mathrm{eV} \mathrm{[78],} \mathrm{where}$ the larger value corresponds to the calculation without SOI. The DFT-1/2 spectrum of $\mathrm{Bi}_{2} \mathrm{O}_{2} \mathrm{Se}$ [Fig. 1(b)] yielding the indirect R- $\Gamma$ gap of $953 \mathrm{meV}$ demonstrates good agreement with both our $\mathrm{mBJ}$ and HSE06 results, with the exception of the $\Gamma-\Gamma$ gap, which is a bit narrower, $1.160 \mathrm{eV}$.

The only experimental value for the band gap in $\mathrm{Bi}_{2} \mathrm{O}_{2} \mathrm{Te}$ was estimated from the maximum of the Seebeck coefficient and was found to be $0.23 \mathrm{eV}$ [52]. The bulk band structure of $\mathrm{Bi}_{2} \mathrm{O}_{2} \mathrm{Te}$ calculated within PBE demonstrates a negative gap [Fig. 1(c)]. The VB maximum lies at the R point at $26 \mathrm{meV}$ above the Fermi level (the VB energy at $\mathrm{X}$ is about $2 \mathrm{meV}$ lower). The CB minimum in the spectrum occurs at the $\mathrm{M}$ point at $203 \mathrm{meV}$ below the Fermi level, while it is $17 \mathrm{meV}$ higher at $\Gamma$. The mBJ exchange potential and HSE06 hybrid functional make the gap positive, whereas they retain relative positions of the gap edge extrema: The VBM is situated at the $\mathrm{R}$ point, and $\mathrm{CBM}$ lies at the $\mathrm{M}$ point, resulting in an indirect R-M gap of 150 and $156 \mathrm{meV}$ in $\mathrm{mBJ}$ and HSE06 calculations, respectively. In the mBJ (HSE06) spectrum the CB minimum at $\Gamma$ lies 125 (36) meV higher than at M. Like in $\mathrm{Bi}_{2} \mathrm{O}_{2} \mathrm{Se}$, with the exception of the extrema points of the spectrum, where good agreement between $\mathrm{mBJ}$ and HSE06 results is observed, HSE06 demonstrates a shift of the bands towards lower (VB) or higher (CB) energies with respect to the mBJ calculation. Earlier $\mathrm{mBJ}$ calculations for $\mathrm{Bi}_{2} \mathrm{O}_{2} \mathrm{Te}$ [76] also demonstrated the R-M indirect gap, the value of which, however, was noticeably larger, $0.21 \mathrm{eV}$, that could be related to larger calculated equilibrium lattice parameters (4.02 and $12.90 \AA$ for $a$ and $c$, respectively). In contrast, in our DFT-1/2 calculation the CBM lies at the $\Gamma$ point, like in $\mathrm{Bi}_{2} \mathrm{O}_{2} \mathrm{Se}$, and the indirect $\mathrm{R}-\Gamma$ gap equals $318 \mathrm{meV}$. This value is two times larger than the mBJ (HSE06) absolute indirect $\mathrm{R}-\mathrm{M}$ gap, and it is larger than the R- $\Gamma$ gap obtained in those calculations (275 meV for $\mathrm{mBJ}$ and $192 \mathrm{meV}$ for HSE06). On the other hand, $G W$ calculations for $\mathrm{Bi}_{2} \mathrm{O}_{2} \mathrm{Te}$ [79] have given the CBM at the $\Gamma$ point and an indirect gap of $340 \mathrm{meV}$ that is very close to our DFT- $1 / 2$ value.

The cutoff radius $r_{c u t}$ in the spherical step function multiplier for the atomic self-energy potential $V_{S}$ is the only parameter of the DFT-1/2 method [71,72] which is determined variationally by maximizing the direct band gap. The dependence of the $\Gamma$ gap on $r_{\text {cut }}$ has typical behavior when varying $\mathrm{O}$ and Bi potentials, demonstrating a maximum within the $r_{\text {cut }}$ range typical of other semiconductors. As was pointed in Ref. [72], often, the self-energy of the cation gives a noticeably smaller contribution to broadening of the gap and can be neglected. Indeed, we found that variation of the Bi potential yields a relatively small gap correction, less than $0.1 \mathrm{eV}$. However, the gap in general diverges with increasing $r_{\text {cut }}$ for the $C h$ atom potential. Using a very short step in $r_{\text {cut }}$, we could find only a toothlike local maximum at $r_{\text {cut }}=2.70$ a.u. for Te [see Fig. 1(d)] and only an inflection point at 2.45 a.u., where the dependence of the $\Gamma$ gap on $r_{\text {cut }}$ changes slope a little bit for Se potential variation. Such a dependence of the band structure on the $C h$ potential parameter does not allow us to apply the DFT-1/2 method confidently for $\mathrm{Bi}_{2} \mathrm{O}_{2} C h$ without knowledge of the experimental data or results of advanced band structure calculation methods. The limitations of the DFT- $1 / 2$ method for transition-metal oxides were also discussed recently [80]. On the other hand, the calculated DFT-1/2 bulk spectra are in close agreement with our mBJ (HSE06) and earlier $G W$ calculation results, which makes this method applicable to surface band structure calculations in $\mathrm{Bi}_{2} \mathrm{O}_{2} \mathrm{Ch}$ compounds.

\section{B. Surface electronic structure}

\section{Unreconstructed polar surfaces}

Recently, nanoplates of $\mathrm{Bi}_{2} \mathrm{O}_{2} \mathrm{Se}$ with a lateral size up to $200 \mu \mathrm{m}$ and thickness varying from monolayer [where 
monolayer (ML) refers to a slab that includes one $\mathrm{Bi}_{2} \mathrm{O}_{2} \mathrm{Se}$ formula unit, i.e., four atomic layers] to tens of nanometers were successfully grown by chemical vapor deposition on a mica substrate [53-55]. These nanoplates are high-quality single crystals with a perfectly square shaped form with atomically flat terraces with (001) orientation and steps with a height of $\sim 0.6 \mathrm{~nm}$, which corresponds to half of the $\mathrm{Bi}_{2} \mathrm{O}_{2} \mathrm{Se}$ $c$ parameter, i.e., 1 ML. HRTEM measurements revealed an ideal unreconstructed atomic arrangement on the terraces.

The $\mathrm{Bi}_{2} \mathrm{O}_{2} C h$ materials are composed of positively charged $\mathrm{Bi}-\mathrm{O}_{2}-\mathrm{Bi}$ trilayers, and $\mathrm{Ch}$ atomic layers have a compensating negative charge. According to our bulk calculations of the Bader charges, we found that $\mathrm{Ch}$ atoms carry about one more electron than its atomic charge, and hence, the $\mathrm{Ch}$ layer negative charge amounts to approximately $-1 e$ (and, correspondingly, it is $\approx+1 e$ on the $\mathrm{Bi}-\mathrm{O}_{2}-\mathrm{Bi}$ trilayer). The values vary a bit with the method of calculation. The Se charge was found to be $-1.20 e,-1.10 e$, and $-1.15 e$ in the $\mathrm{mBJ}$, HSE06, and DFT-1/2, calculations, respectively, and it is $-1.00 e(\mathrm{mBJ}),-0.92 e$ (HSE06), and $-0.99 e$ (DFT-1/2) for the Te atom in $\mathrm{Bi}_{2} \mathrm{O}_{2} \mathrm{Te}$ (hereafter, we will refer only to DFT$1 / 2$ values). Within the $\mathrm{Bi}_{-} \mathrm{O}_{2}-\mathrm{Bi}$ trilayer the $\mathrm{Bader}$ charge on $\mathrm{Bi}$ atoms comprises only $2.20\left(\mathrm{Bi}_{2} \mathrm{O}_{2} \mathrm{Se}\right)$ and $2.26\left(\mathrm{Bi}_{2} \mathrm{O}_{2} \mathrm{Te}\right)$ electrons instead of 5 electrons in the free $\mathrm{Bi}$ atom; that is, $\mathrm{Bi}$ atoms lose almost all of their $p$ electrons in favor of oxygen as well as Ch layer atoms, and consequently, the Bi layer carries a large positive charge. Obviously, under these conditions the formation of the atomically flat single-terminated crystals will lead to the appearance of charged (positive or negative) surfaces depending on surface termination, $\mathrm{Bi}$ or $\mathrm{Ch}$. It is evident such surfaces should demonstrate significantly different work functions and possess distinct surface electronic structures. For our slabs we have calculated work functions of 2.71 and $2.52 \mathrm{eV}$ for $\mathrm{Bi}$ terminations in $\mathrm{Bi}_{2} \mathrm{O}_{2} \mathrm{Se}$ and $\mathrm{Bi}_{2} \mathrm{O}_{2} \mathrm{Te}$, respectively while for the chalcogen atom terminations they are $6.94 \mathrm{eV}\left(\mathrm{Bi}_{2} \mathrm{O}_{2} \mathrm{Se}\right)$ and $6.33 \mathrm{eV}\left(\mathrm{Bi}_{2} \mathrm{O}_{2} \mathrm{Te}\right)$.

Nothing was reported on the surface termination of the grown $\mathrm{Bi}_{2} \mathrm{O}_{2} \mathrm{Se}$ nanoplates in Refs. [53,55]. The Supplementary Information of Ref. [53] reported on the work function measurement for a sample $\sim 5 \mathrm{~nm}$ thick, which revealed a value of $\sim 5.0 \mathrm{eV}$ that varies with the film thickness. This value is close to the average work function calculated for Seand Bi-terminated surfaces. Apparently, at least for general consideration, there is no reason why, with layer-by-layer growth, one termination is preferable to another.

The calculated surface electronic structures for both $\mathrm{Bi}$ and $\mathrm{Ch}$-terminated surfaces in $\mathrm{Bi}_{2} \mathrm{O}_{2} \mathrm{Se}$ and $\mathrm{Bi}_{2} \mathrm{O}_{2} \mathrm{Te}$ are presented in Figs. 2(a)-2(d). In Figs. 2 (e) and 2(f) the electrostatic potential difference $\Delta V=V_{\text {slab }}-V_{\text {bulk }}$, where $V_{\text {slab }}$ and $V_{\text {bulk }}$ are potentials taken at atomic layer planes and integrated over $x y$ for the slab and bulk, respectively, and are connected by curves that serve as a guide to eye for different terminations, $\mathrm{Bi}$ and $\mathrm{Ch}$, of the $\mathrm{Bi}_{2} \mathrm{O}_{2} \mathrm{Ch}$ surfaces is shown. The $\mathrm{Bi}$ terminated surfaces of $\mathrm{Bi}_{2} \mathrm{O}_{2} \mathrm{Se}$ and $\mathrm{Bi}_{2} \mathrm{O}_{2} \mathrm{Te}$, being positively charged, demonstrate negative potential bending [Fig. 2(e)], which smoothly decays to a depth of about 15 atomic layers. In the near-surface layers, except for the smooth negative potential bending, the addition potential oscillations, caused by charge redistribution due to surface relaxation, are observed. This potential bending shifts the Fermi level towards the CB bottom and leads to trapping of electrons of the outermost layers in the surface potential well. The most prominent feature of the surface spectrum of Bi-terminated $\mathrm{Bi}_{2} \mathrm{O}_{2} \mathrm{Se}$ [Fig. 2(a)] is the formation of two surface states in the bulk gap. A pair of parabolic surface states residing at the $\bar{\Gamma}$ point of the spectrum calculated with switched-off SOI [see the right inset in Fig. 2(a)] acquires sizable Rashba spin splitting when the SOI is switched on. Being split off the CB bottom, which is composed of $\mathrm{Bi} p_{z}$ orbitals at $\bar{\Gamma}$, these states have the same orbital character. The lower Rashba state is formed by $p_{z}$ orbitals of the outermost Bi plane [Fig. 3(a)], while the second Rashba-split band is contributed by $p_{z}$ orbitals of the second and third $\mathrm{Bi}$ atomic layers [Fig. 3(b)]. Without taking into account the SOI the $\bar{M}$ surface states are degenerate. The SOI lifts the degeneracy of the $\bar{M}$ states in such a way that one band is raised while another falls, and the resultant two bands at -0.06 and $-0.39 \mathrm{eV}$ have $j=1 / 2$ and $j=3 / 2$ characters, respectively. However, these states are almost spin degenerate near the $\bar{M}$ point. The orbital character of the surface states also explains the negligible spin splitting near the $\bar{M}$ point where these split-off states are determined by the $p_{x, y}$ orbitals of $\mathrm{Bi}$ atoms of the first and second trilayers [Fig. 3(c)], which are less susceptible to the formation of orbital angular momentum, responsible for the formation of Rashba-type surface band splitting [81]. The small energy splitting of the surface bands when $k$ deviates from $\bar{M}$ may also be determined by the hopping integrals between the $\mathrm{Bi}$ $p$ orbitals rather than the strength of SOI, as suggested in Ref. [82]. A similar surface band structure is observed on the Bi-terminated surface of $\mathrm{Bi}_{2} \mathrm{O}_{2} \mathrm{Te}$ [Fig. 2(c)], where, however, due to the smaller gap (and a bit larger potential bending) the lower $\bar{\Gamma}$ Rashba state turns out to be immersed in the bulk VB continuum. The Rashba parameters of the upper $\bar{\Gamma}$ surface state $\left(\alpha_{\mathrm{R}}\right.$ equals $0.34 \mathrm{eV} \AA$ in $\mathrm{Bi}_{2} \mathrm{O}_{2} \mathrm{Se}$ and $0.55 \mathrm{eV} \AA$ in $\mathrm{Bi}_{2} \mathrm{O}_{2} \mathrm{Te}$ ) are about 2.5-3 times larger than those of the lower one $\left(0.15\right.$ and $0.19 \mathrm{eV} \AA$ in $\mathrm{Bi}_{2} \mathrm{O}_{2} \mathrm{Se}$ and $\mathrm{Bi}_{2} \mathrm{O}_{2} \mathrm{Te}$, respectively) in both compounds. These Rashba parameters are much smaller than those calculated for $\mathrm{BiTe} X$ surfaces (1.78$3.50 \mathrm{eV} \AA[8,10,13])$; however, they are comparable to Rashba splitting in $2 \mathrm{D}$ conduction band surface states measured on an aged $\mathrm{Bi}_{2} \mathrm{Se}_{3}$ surface (different fitting procedures for the ARPES-measured bands gave a Rashba splitting parameter varying from 0.36 to $1.35 \mathrm{eV} \AA$ [83]) and those calculated for $\mathrm{Bi}_{2} \mathrm{Se}_{3}\left(0.14-0.24 \mathrm{eV} \AA\right.$ [84]) and $\mathrm{Bi}_{2} \mathrm{Te}_{3}(0.17-0.50 \mathrm{eV} \AA$ [84]) surfaces.

On the $C h$ terminations the surface band structures of $\mathrm{Bi}_{2} \mathrm{O}_{2}$ Se [Fig. 2(b)] and $\mathrm{Bi}_{2} \mathrm{O}_{2}$ Te [Fig. 2(d)] are noticeably different. Although in both cases the surface is negatively charged and in deep layers overall positive potential bending is observed [Fig. 2(f)] for both $\mathrm{Bi}_{2} \mathrm{O}_{2} \mathrm{Se}$ and $\mathrm{Bi}_{2} \mathrm{O}_{2} \mathrm{Te}$, in the outermost layers the behavior of $\Delta V$ is different. In the Teterminated surface of $\mathrm{Bi}_{2} \mathrm{O}_{2}$ Te the potential bending is positive like in the deep layers, whereas $\Delta V$ on the Se-terminated surface of $\mathrm{Bi}_{2} \mathrm{O}_{2} \mathrm{Se}$ demonstrates the opposite slope. The difference in the surface potential bending is related to different surface relaxations of the surface $C h-\mathrm{Bi}$ interlayer spacing, which demonstrates only $2 \%$ contraction for Te and $19 \%$ for the smaller Se atom. The deeper interlayer relaxations are almost the same: The $\mathrm{Bi}-\mathrm{O}$ interlayer relaxation equals $+7 \%$ and $+9 \%$ for $\mathrm{Bi}_{2} \mathrm{O}_{2} \mathrm{Te}$ and $\mathrm{Bi}_{2} \mathrm{O}_{2} \mathrm{Se}$, respectively; the next 


\section{Bi termination}

$\mathrm{Bi}_{2} \mathrm{O}_{2} \mathrm{Se}$

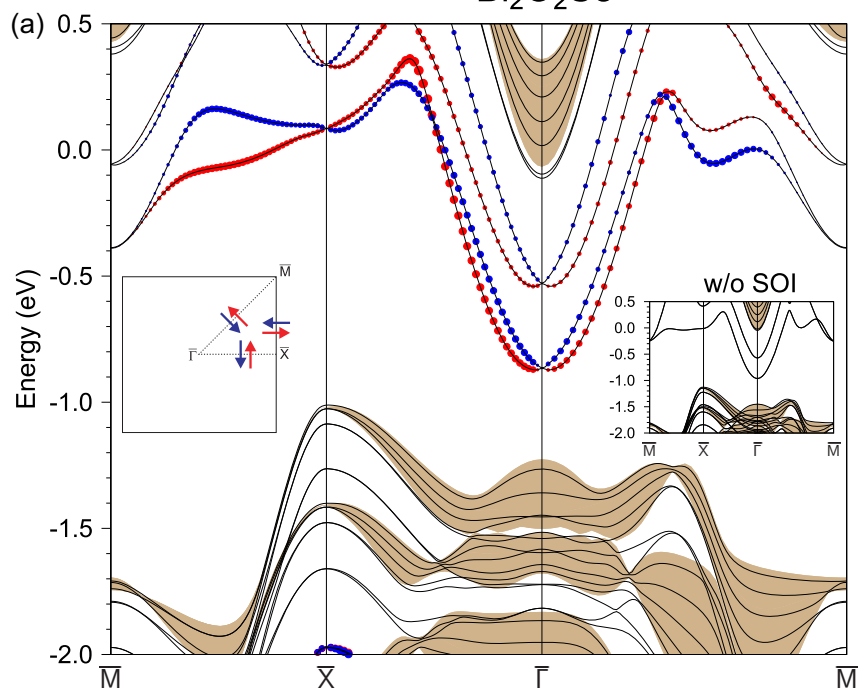

$\mathrm{Bi}_{2} \mathrm{O}_{2} \mathrm{Te}$
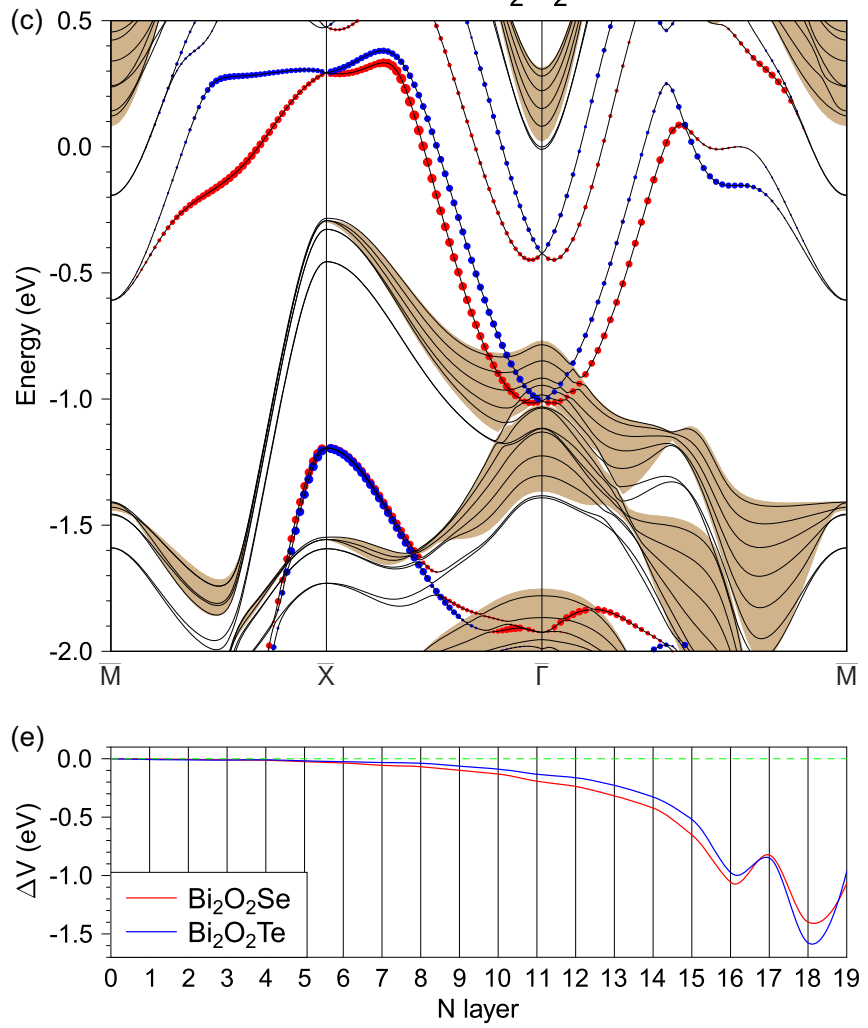

\section{Ch termination}

$\mathrm{Bi}_{2} \mathrm{O}_{2} \mathrm{Se}$

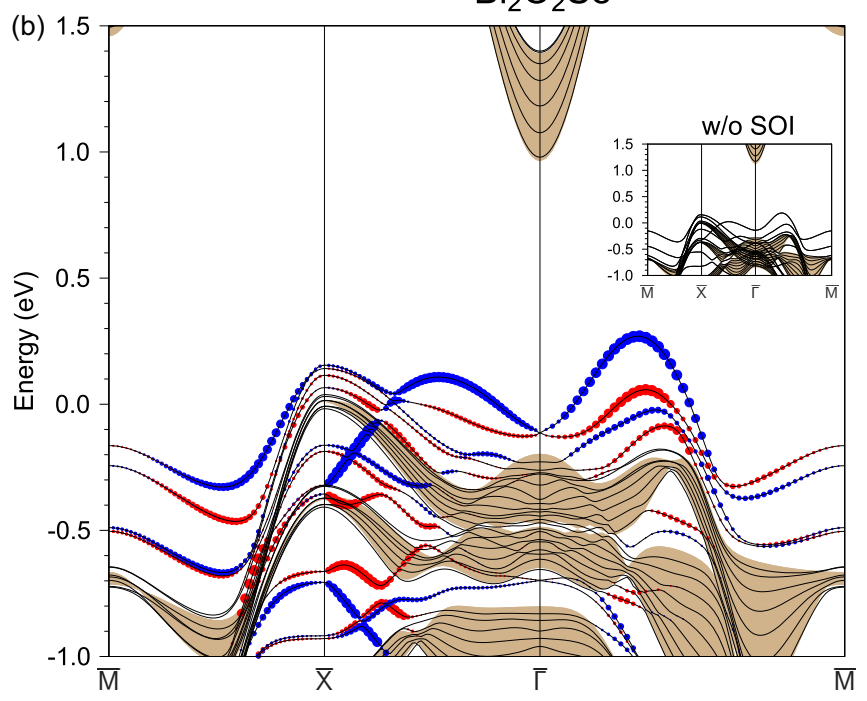

$\mathrm{Bi}_{2} \mathrm{O}_{2} \mathrm{Te}$

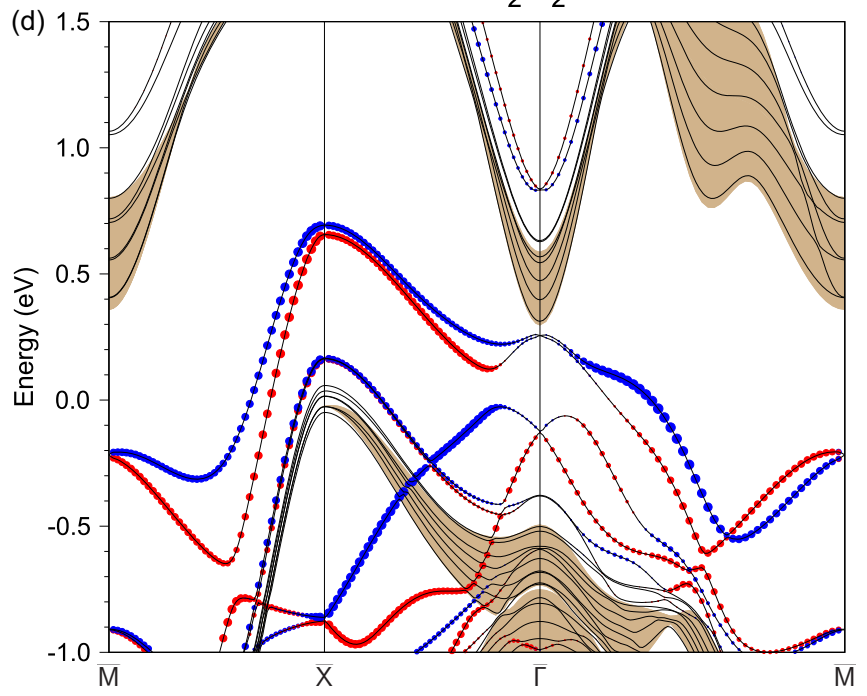

(f)

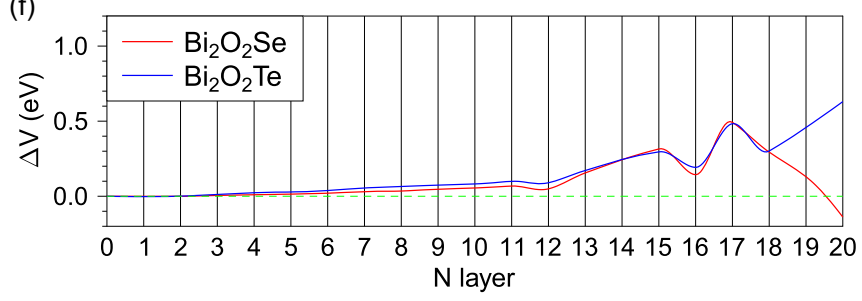

FIG. 2. Surface electronic structure and spin polarization of the polar (a,c) Bi- and (b,d) Ch-terminated surfaces of (a,b) $\mathrm{Bi}_{2} \mathrm{O}_{2} \mathrm{Se}$ and (c,d) $\mathrm{Bi}_{2} \mathrm{O}_{2}$ Te. Red (blue) circles represent the positive (negative) sign of the in-plane spin component for localized states. The left inset in (a) shows the surface Brillouin zone (SBZ) with an indication of directions of positive and negative in-plane spin components for each high-symmetry line of the irreducible part of the SBZ. Right insets in (a) and (b) demonstrate the surface spectra of $\mathrm{Bi}_{2} \mathrm{O}_{2} \mathrm{Se}$ with switched-off SOI. Potential difference $\Delta V=V_{\text {slab }}-V_{\text {bulk }}$ for (e) Bi- and (f) $C h$-terminated surfaces. Zero plane corresponds to the center of the slab.

$\mathrm{O}-\mathrm{Bi}$ interlayer relaxation is $-6 \%$ for both compounds, etc. On the Se-terminated surface of $\mathrm{Bi}_{2} \mathrm{O}_{2} \mathrm{Se}$ a bunch of spin-split localized states appear only in close proximity to the bulk valence band [Fig. 2(b)]. At the $\bar{\Gamma}$ point only one Rashba-like state completely resides in the gap. This state is composed of the $p_{z}$ orbitals of Se atoms of the first layer as well as of the deeper second and third Se layers [Fig. 3(d)], and hence, it penetrates to nine atomic layers. Moving away from the Brillouin zone center towards $\overline{\mathrm{X}}$ and $\overline{\mathrm{M}}$, this state (both spin branches) becomes more localized, and in the middle of the $\bar{\Gamma}-\bar{M}(\bar{X})$ direction it is localized completely within the surface Se layer, acquiring mixed $p_{x y z}$ character [Fig. 3(e)]. On the 

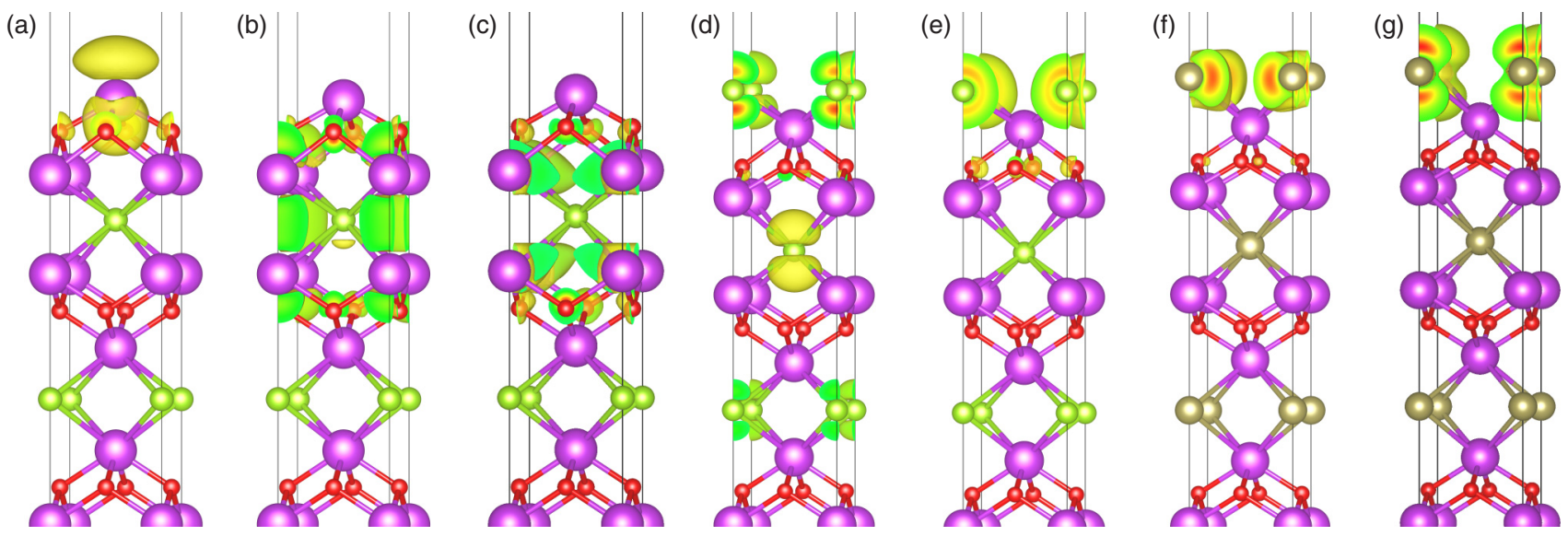

FIG. 3. Charge density distribution of the (a) lower and (b) upper Rashba surface states on the Bi-terminated surface of $\mathrm{Bi}_{2} \mathrm{O}_{2} \mathrm{Se}$ at the $\bar{\Gamma}$ point and (c) the lower state at the $\bar{M}$ point. Charge density of the upper Rashba state on the Se-terminated surface (d) at the $\bar{\Gamma}$ point and (e) in the middle of the $\bar{\Gamma}-\bar{M}$ direction and the charge density distribution on the Te-terminated surface at the (f) $\bar{\Gamma}$ and (g) $\bar{M}$ points.

Te-terminated surface of $\mathrm{Bi}_{2} \mathrm{O}_{2} \mathrm{Te}$ [Fig. 2(d)] the huge positive potential bending splits off a number of surface states from the valence band so that the upper one completely overlies the bulk gap. This state is strongly localized in the outermost Te layer and is determined by the Te $p_{x y}$ orbitals [Fig. 3(f)] at $\bar{\Gamma}$. The lack of $p_{z}$ contribution is responsible for the absence of spin texture at the $\bar{\Gamma}$ point in this case. In contrast, at the $\bar{M}$ point the state is composed solely of $p_{z}$ orbitals of the outermost Te layer [Fig. 3(g)] and demonstrates pronounced spin texture.

\section{Nonpolar $4 \times 1$ reconstructed surfaces}

As shown earlier [53], the Bi-Se interlayer interaction along the $c$ axis is much weaker than the $\mathrm{Bi}-\mathrm{O}$ bonds in the $\mathrm{Bi}-\mathrm{O}_{2}$-Bi trilayers. Hence, the cleavage of the $\mathrm{Bi}_{2} \mathrm{O}_{2} \mathrm{Se}$ crystal must occur in such a way that the atoms of the Se layer will be separated into two new surfaces like in other materials with charged layers $[85,86]$. A certain atomic structure of the cleaved $\mathrm{Bi}_{2} \mathrm{O}_{2}$ Se surface was revealed by the scanning tunneling microscopy measurements [61]. It was demonstrated that the cleavage surface of a single $\mathrm{Bi}_{2} \mathrm{O}_{2} \mathrm{Se}$ crystal shows large, flat terraces with a step height of $\approx 0.61 \mathrm{~nm}(c / 2)$. At the same time each terrace demonstrates a complicated parquetlike surface topography composed of a mixture of perpendicularly oriented (rotationally equivalent) $4 \times 1$ and $1 \times 4$ domains. These domains occur as a result of cleavage when the each half of Se atomic layers is attached to opposite cleavage planes, as required by the charge-neutral conditions, and Se atoms form the rows of dimers. This atomic structure of the $\mathrm{Bi}_{2} \mathrm{O}_{2} \mathrm{Se}$ cleavage surface was corroborated by the DFT total energy calculation and Monte Carlo simulation [61]. The relaxed surface structure of the $\mathrm{Bi}_{2} \mathrm{O}_{2} \mathrm{Se} 4 \times 1$ slab which has 50:50 Se-Bi surface termination is shown in Fig. 4(a). The calculated work function for the nonpolar $\mathrm{Bi}_{2} \mathrm{O}_{2} \mathrm{Se}$ surface is close to the average work functions for corresponding $\mathrm{Bi}$ and Se-terminated surfaces and equals $4.68 \mathrm{eV}(4.82 \mathrm{eV}$ is the average value for work functions of the $\mathrm{Bi}_{2} \mathrm{O}_{2} \mathrm{Se}$ polar surfaces). To explore the nonpolarity of the $4 \times 1$ surface we integrated the slab electrostatic potential separately for the areas with $\mathrm{Bi}$ and $\mathrm{Se}$ terminations and subtracted the bulk potential. As can be seen in Fig. 4(b), unlike polar Bi- and $C h$-terminated surfaces, here for both areas $\Delta V$ in deep layers shows neither positive nor negative bending. In addition, the potential on the outer Se half layer has a value only $0.2 \mathrm{eV}$ less than in the bulk. The largest perturbations in the potential occur in the Bi layer. They are of opposite sign in the Biterminated area and where the $\mathrm{Bi}$ atomic layer lies under the Se dimer, so they nearly completely compensate each other, as clearly seen from the $\Delta V_{\text {tot }}$ behavior.

The surface band structure of the $\mathrm{Bi}_{2} \mathrm{O}_{2} \mathrm{Se}$ surface with mixed Se-Bi termination calculated along the $\overline{\mathrm{Y}}^{\prime}-\bar{\Gamma}-\overline{\mathrm{X}}^{\prime}$ directions of the $4 \times 1$ folded surface Brillouin zone is shown in Fig. 4(c). In contrast to the charged surfaces the spectrum of nonpolar $4 \times 1 \mathrm{Bi}_{2} \mathrm{O}_{2} \mathrm{Se}$ does not possess surface states at the Fermi level. The arising localized spin-split states are mostly located within bulk VB and CB areas. Only two completely occupied surface states lie above the projection of the bulk VB at the $\bar{\Gamma}$ point, and both demonstrate only small Rashba-like splitting. The lower one is localized on the Se dimer row and determined by $p_{x}$ orbitals [Fig. 4(d)], while the upper state is composed primarily of the $p_{y}$ orbitals of Se atoms lying beneath the $\mathrm{Bi}-\mathrm{O}_{2}-\mathrm{Bi}$ trilayer forming the $\mathrm{Bi}$-terminated area of the surface [Fig. 4(e)].

In order to compare the calculated band structure with earlier presented ARPES spectra [53,61] for the $\mathrm{Bi}_{2} \mathrm{O}_{2} \mathrm{Se}$ cleavage surfaces, we have applied to the former a band unfolding technique $[87,88]$ to obtain the band structure along the $\overline{\mathrm{X}}-\bar{\Gamma}-\overline{\mathrm{X}}$ direction of the original $1 \times 1$ surface Brillouin zone (BZ). This unfolded spectrum [Fig. 4(f)] demonstrates a clear resemblance to the experimental ARPES band structure in part of the bulk bands, whereas in the $\bar{\Gamma}$ valley of the VB the only blurring area of significantly lower intensity is visible in the experiment instead of occupied surface states obtained from the calculation. We can attribute this discrepancy to the more complicated surface structure of the real surface, which is a mixture of rotational domains and not a perfect $4 \times 1$ reconstruction.

Constructing the $4 \times 1$ slab for $\mathrm{Bi}_{2} \mathrm{O}_{2} \mathrm{Te}$, which we suppose should have the same structure of the cleavage surface as $\mathrm{Bi}_{2} \mathrm{O}_{2} \mathrm{Se}$, we calculated band structure for its nonpolar surface. Like in the case of the $4 \times 1$ reconstructed $\mathrm{Bi}_{2} \mathrm{O}_{2} \mathrm{Se}$, 


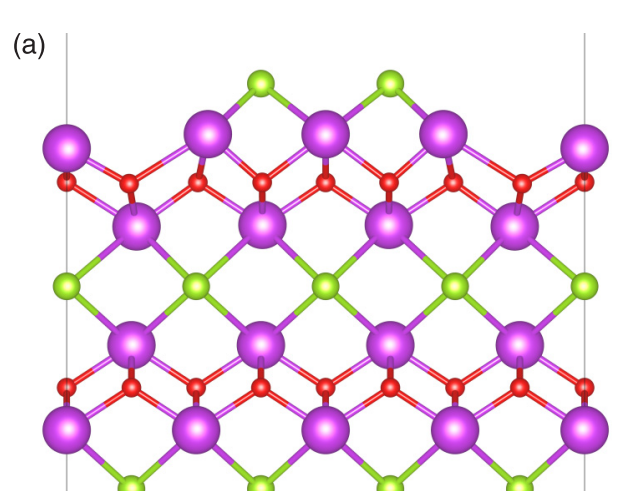

(b)

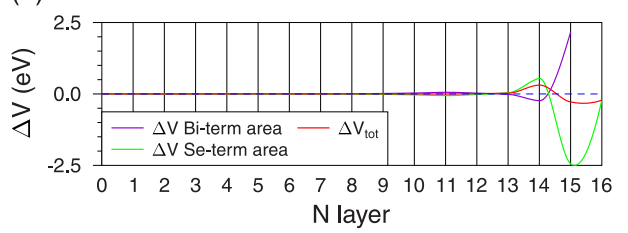

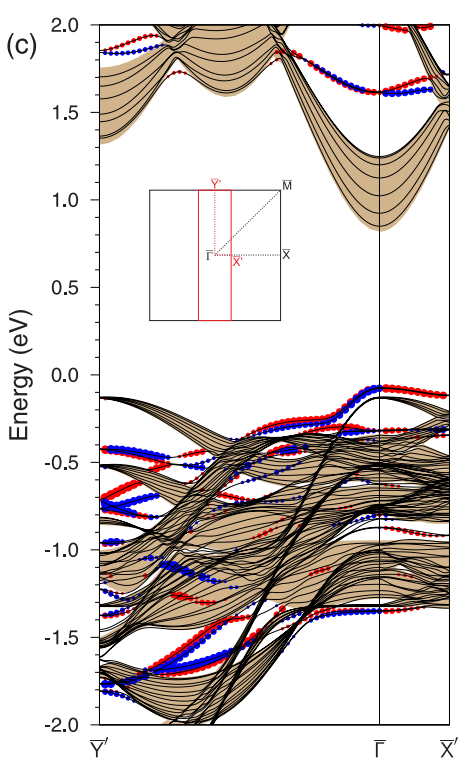

(d)

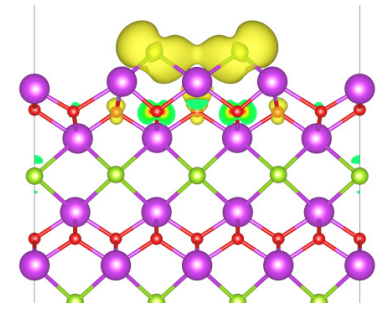

(e)
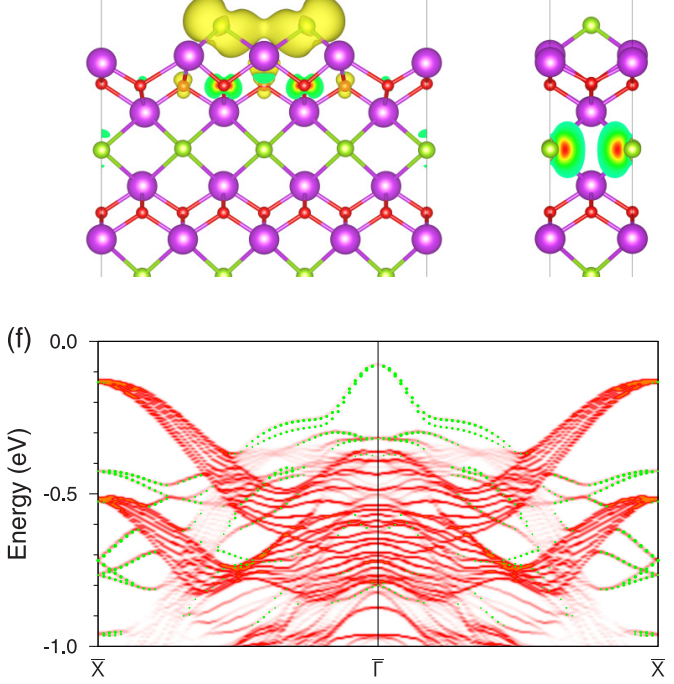

FIG. 4. (a) Side view of the relaxed $4 \times 1$ reconstructed structure of $\mathrm{Bi}_{2} \mathrm{O}_{2} \mathrm{Se}$. (b) $\Delta V$ for Bi- and Se-terminated areas of the nonpolar $\mathrm{Bi}_{2} \mathrm{O}_{2}$ Se surface and $\Delta V_{\text {tot }}$. (c) Spin-resolved surface spectrum of nonpolar $\mathrm{Bi}_{2} \mathrm{O}_{2}$ Se calculated within the $4 \times 1$ Brillouin zone; the inset shows the original $1 \times 1$ and folded $4 \times 1$ BZs (black and red, respectively). Charge density distribution of the (d) lower and (e) upper surface states at the $\bar{\Gamma}$ point. (f) Unfolded spectrum along the $\overline{\mathrm{X}}-\bar{\Gamma}-\overline{\mathrm{X}}$ direction of the $1 \times 1 \mathrm{BZ}$.

the spectrum shows no localized states at the Fermi level and holds two occupied surface states above the VB edge at the $\bar{\Gamma}$ point [Fig. 5(a)] which are best seen in the unfolded spectrum [Fig. 5(c)]. The Rashba splitting in these states is noticeably larger than that of the $4 \times 1 \mathrm{Bi}_{2} \mathrm{O}_{2} \mathrm{Se}$ owing to larger atomic spin-orbit coupling on the heavier Te atom, while their splitting from the VB edge is smaller. The latter is a consequence of weaker disturbance of the surface potential [Fig. 5(b)] than in the case of the $\mathrm{Bi}_{2} \mathrm{O}_{2} \mathrm{Se}$ nonpolar surface.

To illustrate the importance of knowledge of the atomic structure of the cleavage surface we also calculated the $\mathrm{Bi}_{2} \mathrm{O}_{2}$ Te surface spectrum for the slab with $\sqrt{2} \times \sqrt{2}$ reconstruction, where one of two surface Te atoms was removed. Such a reconstruction represents the simplest nonpolar surface with 50:50 Bi-Te surface termination. However, in contrast to the $4 \times 1$ reconstruction, it does not contain $C h$ rows in the
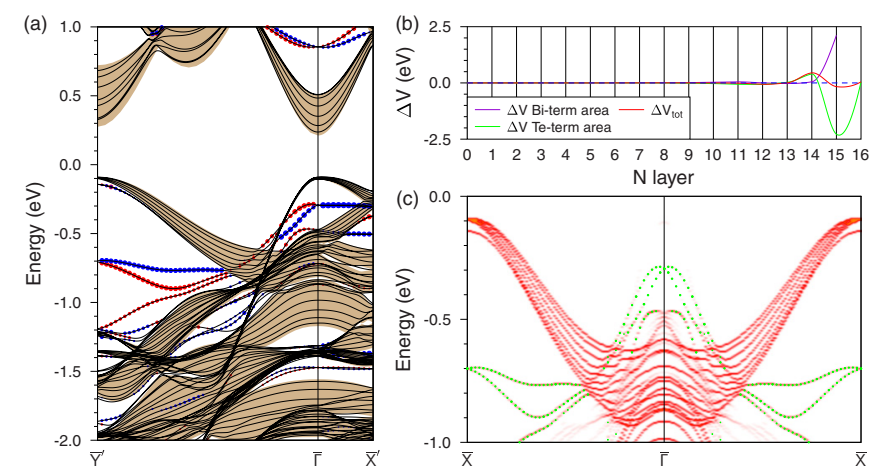

FIG. 5. (a) Spin-resolved surface spectrum of nonpolar $\mathrm{Bi}_{2} \mathrm{O}_{2} \mathrm{Te}$ calculated within the $4 \times 1$ Brillouin zone. (b) $\Delta V$ of the nonpolar $\mathrm{Bi}_{2} \mathrm{O}_{2}$ Te surface. (c) Unfolded spectrum along the $\overline{\mathrm{X}}-\bar{\Gamma}-\overline{\mathrm{X}}$ direction of the $1 \times 1 \mathrm{BZ}$. topmost layer but has isolated $C h$ atoms. The unfolded spectrum along the $\bar{X}-\bar{\Gamma}-\bar{X}$ direction of the $1 \times 1$ surface Brillouin zone is demonstrated in Fig. 6(a). Owing to nonpolarity of the surface, like in the $4 \times 1$ case, there are no metallic states in the spectrum. However, the dispersion of the observed spinsplit states is different. For example, instead of two holelike Rashba states at the $\bar{\Gamma}$ point on the $4 \times 1$ surface, a single almost dispersionless spin-split band arises below the Fermi level. In addition, its orbital symmetry is different. Unlike $p_{x}\left(p_{y}\right)$ surface states on the $4 \times 1$ reconstructed surface, this state is composed of $p_{z}$ orbitals of the outermost isolated Te atoms [Fig. 6(b)]. The geometry of the cleavage surface also affects the work function. On the $4 \times 1$ surface it, like in the $\mathrm{Bi}_{2} \mathrm{O}_{2} \mathrm{Se}$ case, is only a bit smaller than the average work
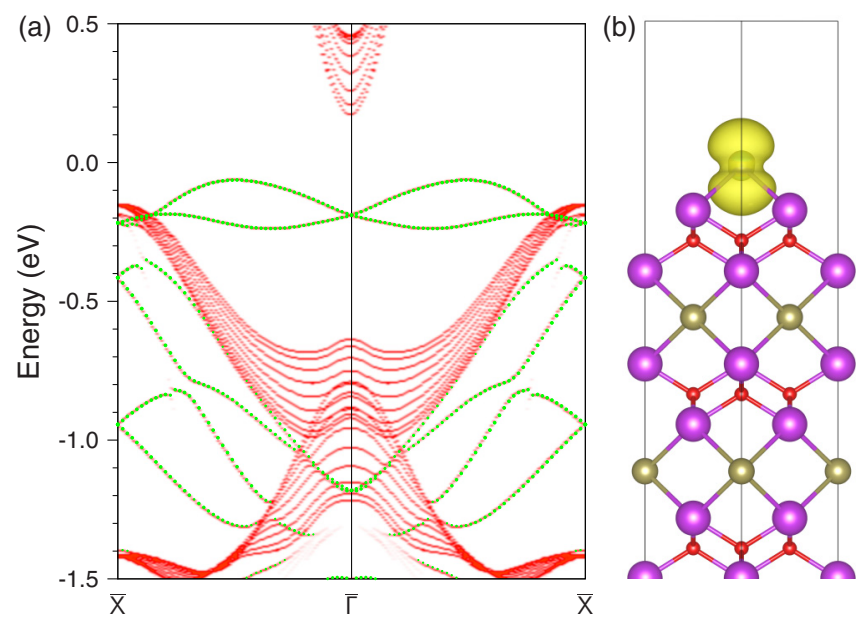

FIG. 6. (a) Unfolded spectrum for nonpolar $\mathrm{Bi}_{2} \mathrm{O}_{2} \mathrm{Te}$ with the $\sqrt{2} \times \sqrt{2}$ reconstruction and (b) charge density distribution of the occupied spin-split surface state at the $\bar{\Gamma}$ point. 
functions for $\mathrm{Bi}$ - and Te-terminated surfaces $(4.50 \mathrm{eV})$, being equal of $4.42 \mathrm{eV}$, whereas on the $\sqrt{2} \times \sqrt{2}$ surface it is much smaller, $4.13 \mathrm{eV}$.

\section{CONCLUSIONS}

In summary, by using first-principles calculations we have examined the surface band structure of the polar unreconstructed $\mathrm{Bi}$ - and $C h$-terminated surfaces of $\mathrm{Bi}_{2} \mathrm{O}_{2} C h$ $(C h=\mathrm{Te}, \mathrm{Se})$, which are realized on nanoplates grown by chemical vapor deposition and nonpolar reconstructed surfaces arising on the cleavage plane of single crystals. We have revealed that both unreconstructed and reconstructed surfaces possess the Rashba-type spin-split surface states. On the polar surfaces, owing to huge surface potential bending, the metallic Rashba states arise in the bulk band gap. These states emerge due to trapping of the valence (on the negatively charged $C h$-terminated surfaces) or conduction (on the positively charged Bi-terminated surfaces) band electrons in the surface potential well. On the nonpolar $\mathrm{Bi}_{2} \mathrm{O}_{2} C h$ cleavage surfaces with mixed $\mathrm{Bi} / \mathrm{Ch}$ termination the deep penetrating surface potential bending is absent, and all emerging surface states are occupied (unoccupied), lying mostly within VB (CB) bulk areas. The dispersion of the surface states depends crucially on the geometry of the nonpolar surface. On the $4 \times$ 1 reconstruction, which was experimentally established as the stable structure for the $\mathrm{Bi}_{2} \mathrm{O}_{2} \mathrm{Ch}(001)$ cleavage surface, two Rashba-split states are located above the bulk VB edge in the $\bar{\Gamma}$ valley of both $\mathrm{Bi}_{2} \mathrm{O}_{2} \mathrm{Se}$ and $\mathrm{Bi}_{2} \mathrm{O}_{2} \mathrm{Te}$, and their Rashba splitting depends on the $C h$ species, being larger for heavier $C h$.

\section{ACKNOWLEDGMENTS}

This work was supported by the Fundamental Research Program of the State Academies of Sciences, line of research III.23, and the Academic D. I. Mendeleev Fund Program of Tomsk State University (Project No. 8.1.01.2018). The support from the Saint Petersburg State University (Project No. 15.61.202.2015), Basque Country Government, Departamento de Educación, Universidades e Investigación (Grant No. IT-756-13), and the Spanish Ministry of Science and Innovation (Grant No. FIS2016-75862-P) is also acknowledged. Calculations were performed using computational resources provided by Resource Center "Computer Center of SPbU" and the SKIF-Cyberia supercomputer at the National Research Tomsk State University. S.V.E. thanks I. A. Nechaev for fruitful discussions.
[1] A. Manchon, H. C. Koo, J. Nitta, S. M. Frolov, and R. A. Duine, Nat. Mater. 14, 871 (2015).

[2] Yu. M. Koroteev, G. Bihlmayer, J. E. Gayone, E. V. Chulkov, S. Blügel, P. M. Echenique, and Ph. Hofmann, Phys. Rev. Lett. 93, 046403 (2004).

[3] C. R. Ast, J. Henk, A. Ernst, L. Moreschini, M. C. Falub, D. Pacilé, P. Bruno, K. Kern, and M. Grioni, Phys. Rev. Lett. 98, 186807 (2007).

[4] G. Bihlmayer, S. Blügel, and E. V. Chulkov, Phys. Rev. B 75, 195414 (2007)

[5] C. R. Ast, D. Pacilé, L. Moreschini, M. C. Falub, M. Papagno, K. Kern, M. Grioni, J. Henk, A. Ernst, S. Ostanin, and P. Bruno, Phys. Rev. B 77, 081407(R) (2008).

[6] H. Mirhosseini, J. Henk, A. Ernst, S. Ostanin, C.-T. Chiang, P. Yu, A. Winkelmann, and J. Kirschner, Phys. Rev. B 79, 245428 (2009).

[7] K. Ishizaka, M. S. Bahramy, H. Murakawa, M. Sakano, T. Shimojima, T. Sonobe, K. Koizumi, S. Shin, H. Miyahara, A. Kimura, K. Miyamoto, T. Okuda, H. Namatame, M. Taniguchi, R. Arita, N. Nagaosa, K. Kobayashi, Y. Murakami, R. Kumai, Y. Kaneko, Y. Onose, and Y. Tokura, Nat. Mater. 10, 521 (2011).

[8] S. V. Eremeev, I. A. Nechaev, Yu. M. Koroteev, P. M. Echenique, and E. V. Chulkov, Phys. Rev. Lett. 108, 246802 (2012)

[9] I. P. Rusinov, I. A. Nechaev, S. V. Eremeev, C. Friedrich, S. Blügel, and E. V. Chulkov, Phys. Rev. B 87, 205103 (2013).

[10] S. V. Eremeev, I. P. Rusinov, I. A. Nechaev, and E. V. Chulkov, New J. Phys. 15, 075015 (2013).

[11] A. Crepaldi, L. Moreschini, G. Autès, C. Tournier-Colletta, S. Moser, N. Virk, H. Berger, Ph. Bugnon, Y. J. Chang, K. Kern, A. Bostwick, E. Rotenberg, O. V. Yazyev, and M. Grioni, Phys. Rev. Lett. 109, 096803 (2012).
[12] G. Landolt, S. V. Eremeev, Yu. M. Koroteev, B. Slomski, S. Muff, T. Neupert, M. Kobayashi, V. N. Strocov, T. Schmitt, Z. S. Aliev, M. B. Babanly, I. R. Amiraslanov, E. V. Chulkov, J. Osterwalder, and J. H. Dil, Phys. Rev. Lett. 109, 116403 (2012).

[13] S. V. Eremeev, I. A. Nechaev, and E. V. Chulkov, JETP Lett. 96, 437 (2012).

[14] G. Landolt, S. V. Eremeev, O. E. Tereshchenko, S. Muff, B. Slomski, K. A. Kokh, M. Kobayashi, T. Schmitt, V. N. Strocov, J. Osterwalder, E. V. Chulkov, and J. H. Dil, New J. Phys. 15, 085022 (2013).

[15] M. Sakano, M. S. Bahramy, A. Katayama, T. Shimojima, H. Murakawa, Y. Kaneko, W. Malaeb, S. Shin, K. Ono, H. Kumigashira, R. Arita, N. Nagaosa, H. Y. Hwang, Y. Tokura, and K. Ishizaka, Phys. Rev. Lett. 110, 107204 (2013).

[16] N. Ogawa, M. S. Bahramy, H. Murakawa, Y. Kaneko, and Y. Tokura, Phys. Rev. B 88, 035130 (2013).

[17] C. Tournier-Colletta, G. Autès, B. Kierren, Ph. Bugnon, H. Berger, Y. Fagot-Revurat, O. V. Yazyev, M. Grioni, and D. Malterre, Phys. Rev. B 89, 085402 (2014).

[18] S. Fiedler, T. Bathon, S. V. Eremeev, O. E. Tereshchenko, K. A. Kokh, E. V. Chulkov, P. Sessi, H. Bentmann, M. Bode, and F. Reinert, Phys. Rev. B 92, 235430 (2015).

[19] H. Maaß, H. Bentmann, C. Seibel, C. Tusche, S. V. Eremeev, T. R. F. Peixoto, O. E. Tereshchenko, K. A. Kokh, E. V. Chulkov, J. Kirschner, and F. Reinert, Nat. Commun. 7, 11621 (2016).

[20] M. Z. Hasan and C. L. Kane, Rev. Mod. Phys. 82, 3045 (2010).

[21] M. Zahid Hasan and J. E. Moore, Annu. Rev. Condens. Matter Phys. 2, 55 (2011).

[22] A. Bansil, H. Lin, and T. Das, Rev. Mod. Phys. 88, 021004 (2016). 
[23] I. A. Nechaev, S. V. Eremeev, E. E. Krasovskii, P. M. Echenique, and E. V. Chulkov, Sci. Rep. 7, 43666 (2017).

[24] S. V. Eremeev, I. A. Nechaev, and E. V. Chulkov, Phys. Rev. B 96, 155309 (2017).

[25] M. K. Tran, J. Levallois, P. Lerch, J. Teyssier, A. B. Kuzmenko, G. Autès, O. V. Yazyev, A. Ubaldini, E. Giannini, D. van der Marel, and A. Akrap, Phys. Rev. Lett. 112, 047402 (2014).

[26] I. P. Rusinov, T. V. Menshchikova, I. Yu. Sklyadneva, R. Heid, K.-P. Bohnen, and E. V. Chulkov, New J. Phys. 18, 113003 (2016).

[27] S. Fiedler, S. V. Eremeev, V. A. Golyashov, A. K. Kaveev, O. E. Tereshchenko, K. A. Kokh, E. V. Chulkov, H. Bentmann, and F. Reinert, New J. Phys. 20, 063035 (2018).

[28] Y. Xia, D. Qian, D. Hsieh, L. Wray, A. Pal, H. Lin, A. Bansil, D. Grauer, Y. S. Hor, R. J. Cava, and M. Z. Hasan, Nat. Phys. 5, 398 (2009).

[29] Y. L. Chen, J. G. Analytis, J.-H. Chu, Z. K. Liu, S.-K. Mo, X. L. Qi, H. J. Zhang, D. H. Lu, X. Dai, Z. Fang, S. C. Zhang, I. R. Fisher, Z. Hussain, and Z.-X. Shen, Science 325, 178 (2009).

[30] T. Zhang, P. Cheng, X. Chen, J.-F. Jia, X. Ma, K. He, L. Wang, H. Zhang, X. Dai, Z. Fang, X. Xie, and Q.-K. Xue, Phys. Rev. Lett. 103, 266803 (2009).

[31] K. Kuroda, M. Arita, K. Miyamoto, M. Ye, J. Jiang, A. Kimura, E. E. Krasovskii, E. V. Chulkov, H. Iwasawa, T. Okuda, K. Shimada, Y. Ueda, H. Namatame, and M. Taniguchi, Phys. Rev. Lett. 105, 076802 (2010).

[32] Y. Zhang, K. He, C.-Z. Chang, C.-L. Song, L.-L. Wang, X. Chen, J.-F. Jia, Z. Fang, X. Dai, W.-Y. Shan, S.-Q. Shen, Q. Niu, X.-L. Qi, S.-C. Zhang, X.-C. Ma, and Q.-K. Xue, Nat. Phys. 6, 584 (2010).

[33] J.-H. Song, H. Jin, and A. J. Freeman, Phys. Rev. Lett. 105, 096403 (2010).

[34] W. Zhang, R. Yu, H.-J. Zhang, X. Dai, and Z. Fang, New J. Phys. 12, 065013 (2010).

[35] S. V. Eremeev, Yu. M. Koroteev, and E. V. Chulkov, JETP Lett. 91, 387 (2010).

[36] L.-L. Wang, and D. D. Johnson, Phys. Rev. B 83, 241309(R) (2011).

[37] Z. Ren, A. A. Taskin, S. Sasaki, K. Segawa, and Y. Ando, Phys. Rev. B 82, 241306(R) (2010).

[38] H. Lin, T. Das, L. A. Wray, S.-Y. Xu, and M. Z. Hasan, and A. Bansil, New J. Phys. 13, 095005 (2011).

[39] E. Annese, T. Okuda, E. F. Schwier, H. Iwasawa, K. Shimada, M. Natamane, M. Taniguchi, I. P. Rusinov, S. V. Eremeev, K. A. Kokh, V. A. Golyashov, O. E. Tereshchenko, E. V. Chulkov, and A. Kimura, Phys. Rev. B 97, 205113 (2018).

[40] X. Ma, D. Chang, C. Zhao, R. Li, X. Huang, Z. Zeng, X. Huang, and Y. Jia, J. Mater. Chem. C 6, 13241 (2018).

[41] L. Li, Y. Liu, J. Dai, H. Zhu, A. Hong, X. Zhou, Z. Ren, and J. Liu, Nano Energy 12, 447 (2015).

[42] E. Koyama, I. Nakai, and K. Nagashima, Acta Crystallogr., Sect. B 40, 105 (1984).

[43] H. Boller, Monatsh. Chem. 104, 916 (1973).

[44] C. Drasar, P. Ruleova, L. Benes, and P. Lostak, J. Electron. Mater. 41, 2317 (2012).

[45] P. Schmidt, O. Rademacher, and H. Opperman, Z. Anorg. Allg. Chem. 625, 255 (1999).
[46] P. Schmidt, O. Bosholm, and H. Oppermann, Z. Naturforsch. B 52, 1461 (1997).

[47] P. Schmidt, O. Rademacher, H. Oppermann, and S. Däbritz, Z. Anorg. Allg. Chem. 626, 1999 (2000).

[48] P. Ruleova, C. Drasar, P. Lostak, C.-P. Li, S. Ballikaya, and C. Uher, Mater. Chem. Phys. 119, 299 (2010).

[49] K. Zhang, C. Hu, Z. Kang, S. Wang, Y. Xi, and H. Liu, Mater. Res. Bull. 48, 3968 (2013).

[50] T. V. Quang, H. Lim, and M. Kim, J. Korean Phys. Soc. 61, 1728 (2012).

[51] D. Guo, C. Hu, Y. Xi, and K. Zhang, J. Phys. Chem. C 117, 21597 (2013).

[52] S. D. N. Luu and P. Vaqueiro, J. Solid State Chem. 226, 219 (2015).

[53] J. Wu, H. Yuan, M. Meng, C. Chen, Y. Sun, Z. Chen, W. Dang, C. Tan, Y. Liu, J. Yin, Y. Zhou, S. Huang, H. Q. Xu, Y. Cui, H. Y. Hwang, Z. Liu, Y. Chen, B. Yan, and H. Peng, Nat. Nanotechnol. 12, 530 (2017).

[54] J. Wu, C. Tan, Z. Tan, Y. Liu, J. Yin, W. Dang, M. Wang, and H. Peng, Nano Lett. 17, 3021 (2017).

[55] M. Meng, S. Huang, C. Tan, J. Wu, Y. Jing, H. Peng, and H. Q. $\mathrm{Xu}$, Nanoscale 10, 2704 (2018).

[56] X. Tian, H. Luo, R. Wei, C. Zhu, Q. Guo, D. Yang, F. Wang, J. Li, J. Qiu, Adv. Mater. 30, 1801021 (2018).

[57] T. Tong, M. Zhang, Y. Chen, Y. Li, L. Chen, J. Zhang, F. Song, X. Wang, W. Zou, Y. Xu, and R. Zhang, Appl. Phys. Lett. 113, 072106 (2018).

[58] J. Wu, C. Qiu, H. Fu, S. Chen, C. Zhang, Z. Dou, C. Tan, T. Tu, T. Li, Y. Zhang, Z. Zhang, L.-M. Peng, P. Gao, B. Yan, and H. Peng, Nano Lett. 19, 197 (2019).

[59] Q. Fu, C. Zhu, X. Zhao, X. Wang, A. Chaturvedi, C. Zhu, X. Wang, Q. Zeng, J. Zhou, F. Liu, B. K. Tay, H. Zhang, S. J. Pennycook, and Z. Liu, Adv. Mater. 31, 1804945 (2019).

[60] J. Yin, Z. Tan, H. Hong, J. Wu, H. Yuan, Y. Liu, C. Chen, C. Tan, F. Yao, T. Li, Y. Chen, Z. Liu, K. Liu, and H. Peng, Nat. Commun. 9, 3311 (2018).

[61] C. Chen, M. Wang, J. Wu, H. Fu, H. Yang, Z. Tian, T. Tu, H. Peng, Y. Sun, X. Xu, J. Jiang, N. B. M. Schröter, Y. Li, D. Pei, S. Liu, S. A. Ekahana, H. Yuan, J. Xue, G. Li, J. Jia, Z. Liu, B. Yan, H. Peng, and Y. Chen, Sci. Adv. 4, eaat8355 (2018).

[62] G. Kresse and J. Hafner, Phys. Rev. B 48, 13115 (1993).

[63] G. Kresse and J. Furthmüller, Phys. Rev. B 54, 11169 (1996).

[64] P. E. Blöchl, Phys. Rev. B 50, 17953 (1994).

[65] G. Kresse and D. Joubert, Phys. Rev. B 59, 1758 (1999).

[66] J. P. Perdew, K. Burke, and M. Ernzerhof, Phys. Rev. Lett. 77, 3865 (1996).

[67] S. Grimme, J. Antony, S. Ehrlich, and H. Krieg, J. Chem. Phys. 132, 154104 (2010).

[68] A. D. Becke and E. R. Johnson, J. Chem. Phys. 124, 221101 (2006).

[69] F. Tran and P. Blaha, Phys. Rev. Lett. 102, 226401 (2009).

[70] A. V. Krukau, O. A. Vydrov, A. F. Izmaylov, and G. E. Scuseria, J. Chem. Phys. 125, 224106 (2006).

[71] L. G. Ferreira, M. Marques, and L. K. Teles, Phys. Rev. B 78, 125116 (2008).

[72] L. G. Ferreira, M. Marques, and L. K. Teles, AIP Adv. 1, 32119 (2011).

[73] K. Momma and F. Izumi, J. Appl. Crystallogr. 44, 1272 (2011). 
[74] A. L. J. Pereira, D. Santamaria-Peréz, J. Ruiz-Fuertes, F. J. Manjón, V. P. Cuenca-Gotor, R. Vilaplana, O. Gomis, C. Popescu, A. Munoz, P. Rodriguez-Hernandez, A. Segura, L. Gracia, A. Flors Beltran, P. Ruleova, C. Drasar, and J. A. Sans Tresserras, J. Phys. Chem. C 122, 8853 (2018).

[75] Y. Wang, B. Xu, G. Yu, J. Zhang, S. Ma, S. Yuan, T. Sun, and Y. Wang, Jpn. J. Appl. Phys. 58, 015501 (2019).

[76] C. Wang, G. Ding, X. Wu, S. Wei, and G. Gao, New J. Phys. 20, 123014 (2018).

[77] M. Wu and X. C. Zeng, Nano Lett. 17, 6309 (2017).

[78] J. Liu, L. Tian, Y. Mou, W. Jia, L. Zhang, and R. Liu, J. Alloys Compd. 764, 674 (2018).

[79] Q. Wei, C. Lin, Y. Li, X. Zhang, Q. Zhang, Q. Shen, Y. Cheng, and W. Huang, J. Appl. Phys. 124, 055701 (2018).

[80] J. Doumont, F. Tran, and P. Blaha, Phys. Rev. B 99, 115101 (2019).

[81] S. R. Park, C. H. Kim, J. Yu, J. H. Han, and C. Kim, Phys. Rev. Lett. 107, 156803 (2011).

[82] H. Ishida, Phys. Rev. B 98, 205412 (2018).
[83] P. D. C. King, R. C. Hatch, M. Bianchi, R. Ovsyannikov, C. Lupulescu, G. Landolt, B. Slomski, J. H. Dil, D. Guan, J. L. Mi, E. D. L. Rienks, J. Fink, A. Lindblad, S. Svensson, S. Bao, G. Balakrishnan, B. B. Iversen, J. Osterwalder, W. Eberhardt, F. Baumberger, and Ph. Hofmann, Phys. Rev. Lett. 107, 096802 (2011).

[84] S. V. Eremeev, M. G. Vergniory, T. V. Menshchikova, A. A. Shaposhnikov, and E. V. Chulkov, New J. Phys. 14, 113030 (2012).

[85] F. Ostendorf, C. Schmitz, S. Hirth, A. Kuhnle, J. J. Kolodziej, and M. Reichling, Nanotechnology 19, 305705 (2008).

[86] K. Kuroda, M. Ye, E. F. Schwier, M. Nurmamat, K. Shirai, M. Nakatake, S. Ueda, K. Miyamoto, T. Okuda, H. Namatame, M. Taniguchi, Y. Ueda, and A. Kimura, Phys. Rev. B 88, 245308 (2013).

[87] P. V. C. Medeiros, S. Stafström, and J. Björk, Phys. Rev. B 89, 041407(R) (2014).

[88] P. V. C. Medeiros, S. S. Tsirkin, S. Stafström, and J. Björk, Phys. Rev. B 91, 041116(R) (2015). 